\title{
Clinical Performance of Two Types of Primary Molar Indirect Crowns Fabricated by 3D Printer and CAD/CAM for Rehabilitation of Large Carious Primary Molars
}

\author{
Mohammed Nour Al-Halabi ${ }^{1} \quad$ Nada Bshara ${ }^{1} \quad$ Jihad Abou Nassar ${ }^{2} \quad$ John C. Comisi, ${ }^{3, \odot ~ C h a r l i n e ~ K . ~ R i z k ~}{ }^{4}$
}

${ }^{1}$ Pediatric Dentistry Department, Dental College, Damascus University, Al-Mazzeh Street, Damascus, Syria

2Department of Fixed Prosthodontics, Dental College, Damascus University, Damascus, Syria

${ }^{3}$ Department of Oral Rehabilitation, James B. Edwards College of Dental Medicine, Medical University of South Carolina, Charleston, South Carolina, United States

${ }^{4}$ Department of Pediatric Dentistry and Dental Public Health, Lebanese University, School of Dentistry, Beirut, Lebanon

\author{
Address for correspondence John C. Comisi, DDS, Department of \\ Oral Rehabilitation, James B Edwards College of Dental Medicine, \\ Medical University of South Carolina, 173 Ashely Avenue BSB \\ 548 MSC 507, Charleston, SC 29425, United States \\ (e-mail:comisi@musc.edu).
}

Eur J Dent 2021;15:463-468

\begin{abstract}
Objectives This randomized clinical trial aimed to evaluate clinical outcomes of two types of esthetic crowns fabricated using a three-dimensional (3D) dental printer and computer-aided design and manufacturing (CAD/CAM) system as an alternative full-coronal restoration for extensively carious pulp-treated primary molars.

Materials and Methods Randomization was done for 50 lower primary molars in 50 child patients, split into two groups based on the fabrication method used: Group A: CAD/CAM crowns using polymethyl methacrylate (PMMA) blocks and Group B: 3D dental printed crowns using GC photopolymer resin. All crowns were evaluated at baseline and at 3rd, 6th, and 12th months using the U.S. Public Health Service criteria for gingival health, retention, and marginal integrity for both groups.

Statistical Analysis The survival rate of 3D-printable crowns was $84 \%$ compared with $80 \%$ survival rate using CAD/CAM fabricated crowns at the end of the 12 th-month follow-up. No statistically significant differences were noted in restoration failure. In the evaluation of gingival health between the two groups' follow-up times, no statistically significant differences were noted at the 3rd- and 6th-month follow-ups, yet at the 12 th month, statistically significant differences were noted $(p=0.022)$ when comparing gingival health. No statistically significant differences were noted between the two

Keywords

-3D dental printer

- CAD/CAM

- posterior

- crowns

- primary teeth groups when comparing marginal integrity scores in all the follow-up periods.

Conclusion Resin crowns fabricated via 3D dental printer and PMMA crowns fabricated using CAD/CAM are acceptable esthetic choices in restoring pulp-treated primary molars with great marginal integrity and crowns retention. 3D-printed resin crowns showed less cementing failure and performed better regarding gingival response compared with PMMA crowns.
\end{abstract}

published online

February 3, 2021
DoI https://doi.org/

10.1055/s-0040-1721905

ISSN 1305-7456. (c) 2021. European Journal of Dentistry.

This is an open access article published by Thieme under the terms of the Creative Commons Attribution-NonDerivative-NonCommercial-License, permitting copying and reproduction so long as the original work is given appropriate credit. Contents may not be used for commercial purposes, or adapted, remixed, transformed or built upon. (https://creativecommons.org/licenses/by-nc-nd/4.0/)

Thieme Medical and Scientific Publishers Pvt. Ltd. A-12, 2nd Floor, Sector 2, Noida-201301 UP, India 


\section{Introduction}

Management of primary teeth caries in patients with high caries risk is always a challenge for pediatric dentists. Stainless steel crowns (SSCs) are the gold standard in restoring pulpotomized primary molars because of their advantage in maintaining morphologic form and superior clinical performance. ${ }^{1}$ However, the limitation of their use is the metallic appearance, which is becoming unacceptable in today's modern world. Additionally, parents' expectations for the recovery of their children's carious teeth and the growing need for esthetic treatment ${ }^{2}$ have expanded the need for alternative esthetic crowns, especially in patients with early childhood caries. $^{3}$

Open-faced stainless-steel crowns have been mentioned in the literature as an aesthetic alternative for traditional SSC; however, they can provide an unfavorable bulky semblance and poor clinical performance. ${ }^{4}$ Preveneer SSCs have been used; however, their expense and the absence of a natural appearance have been a significant drawback and, as such, limited its usage. ${ }^{5}$ Recently, prefabricated zirconia crowns have shown a great advantage as a replacement for SSC in restoring pulpotomized primary molars, with high esthetic and clinical performance. ${ }^{6}$ Nevertheless, the lack of long-term evidence of their benefit and high cost has limited its usage, especially with children living in poverty, who suffer twice as much tooth decay than affluent children who have limited clinical use of ready-made zirconia crowns. ${ }^{7}$

In recent years, digital dentistry has rapidly developed, especially in three-dimensional dental printing and computer-aided design and manufacturing (CAD/CAM) manufacturing with different types of biocompatible restoration materials. ${ }^{8,9}$

CAD/CAM imaging and milling systems have made immense improvement through the recent decade. This technology can create perfect occlusal and proximal contact points with ideal marginal integrity. ${ }^{10}$ These CAD/CAM devices are becoming readily available even in private offices and dental clinics in addition to their use in dental technician laboratories, with different types of materials available, including ceramic, zirconia, resin, and polymethyl methacrylate (PMMA) blocks, which had excellent mechanical properties. ${ }^{11}$

Moreover, the most recent digital dentistry development revolves around the 3D printing technology (3D printer), which has made an impactful entrance in clinical dentistry. ${ }^{8}$ Printed crowns and bridges using digital light processing (DLP) systems via 3D printer have been shown to have mechanical properties for intraoral use as durable provisional and long-term restoration. ${ }^{12}$

Therefore, this randomized clinical trial's overarching objective was to investigate clinical outcomes of two low-cost alternative esthetic crowns fabricated using a 3D dental printer and CAD/CAM system as a full-coronal restoration for extensively carious pulp-treated primary molars. To the best of our knowledge, this is the first randomized clinical trial to compare the photopolymer resin via 3D printing system and PMMA blocks through CAD/CAM used in fabricating the restoration of primary molars, and this trial is registered at ANZCTR under registration number (trial ID: ACTRN12619001650189).

\section{Materials and Methods}

\section{Study Design}

The study design was double-blind, parallel groups, randomized clinical trial following the Consolidated Standards of Reporting Trials protocol (-Fig. 1). ${ }^{13}$

\section{Participant Recruitment}

The study was conducted over 3 months, with a 12-month follow-up from April 2019 to August 2019 to August 2020. Children enrolled in this study aged between 4 and 8 years (6.04 mean age) were selected from treated patients at the department of pediatric dentistry, of the lead authors' university, based on the following inclusion criteria:

- Healthy children without any mental problems or systemic disorders

- Cooperative patients with (definitely positive-positive) ratings on the Frank rating scale. ${ }^{14}$

- A patient who needed pulp treatment of carious primary molars indicated for full coverage with two affected surfaces.

Children with (negative-definitely negative) Frank behavior classification scale were excluded, and teeth with abscess/fistula or discoloration related to tooth nonvitality were excluded.

Prior to enrollment, written informed consent that described the purpose of the research was distributed to the children's parents or guardians of the eligible children. Once completed, the children were enrolled.

\section{Sample Size}

The sample size calculation was calculated using $\mathrm{G}^{*}$ Power 3.1.9 (Franz Faul, Universität Kiel), 0.05 significance level, and $80 \%$ statistical power. It was estimated that 22 crowns in each group were required, ${ }^{15}$ yet the total sample size was raised to 25 crowns to avoid the possible negative effect drop rate.

\section{Clinical Procedure}

A total of 50 teeth from 50 child patients were randomly allocated into one of the following groups:

Group A: Selected teeth crowned with PMMA-based crowns fabricated using CAD/CAM.

Group B: Selected teeth crowned using 3D printable biocompatible light-curing composite crowns fabricated using a 3D dental printer.

After the clinical and radiographic examination of the selected teeth, one operator completed all treatment procedures, using local anesthesia (lidocaine hydrochloride 2\% with epinephrine $1: 100,000)$, rubber dam isolation, removal of caries, pulpotomy, then the restoration of the remaining tooth structure with glass ionomer cement (Fuji IX, GC, Tokyo, Japan).

Tooth crowning was assigned to a specific group according to the applied crown type, 25 teeth crowned with 


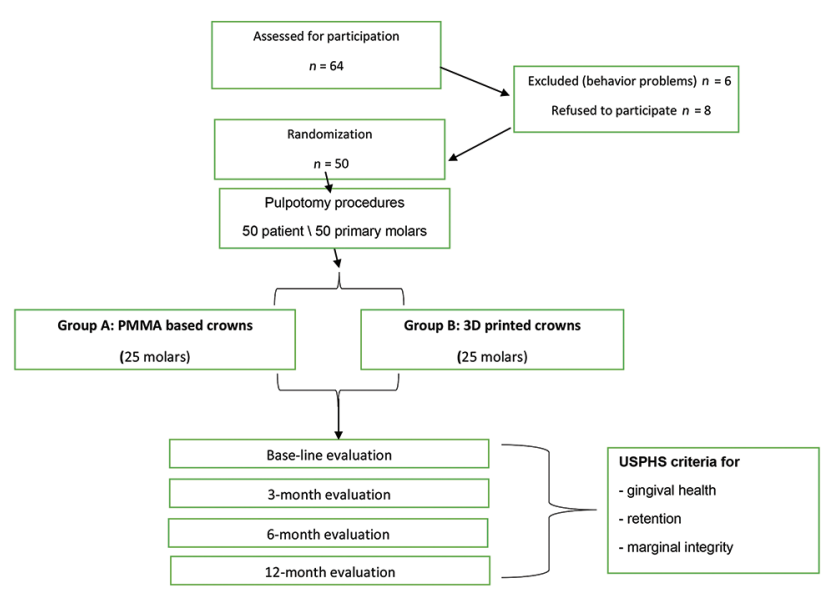

Fig. 1 Consolidated Standards of Reporting Trials diagram for the trial. PMMA, polymethyl methacrylate; USPHS, U.S. Public Health Service.

photopolymer resin-based material fabricated using a 3D dental printer in group A, and 25 teeth crowned with PPMAbased material fabricated using CAD/CAD system.

The selected teeth were prepared with the diamond bur round end taper for buccal, lingual, mesial, and distal wall preparation of 0.8 to $1.0 \mathrm{~mm}$, followed by a chamfer margin circumferentially, and then, the occlusal surface was reduced, leaving a gap of $1.5 \mathrm{~mm}$ with the opposing tooth using a round wheel (Komet, Brasseler, Lemgo, Germany) with a convergence angle of 6 degrees which is maintained by holding the burr parallel to the vertical axis of the tooth.

A polyvinylsiloxane impression (Examix NDS, GC Corporation, Tokyo, Japan) was made for the prepared teeth, and then, an alginate impression was also created for the opposite jaw.

Digital scanning for the dye creation using Medit T300 3D Dental Scanners (MEDIT corp. 23 Goryeodae-ro 22-gil, Seongbuk-gu, Seoul, Korea) was accomplished. The crowns were designed for full coronal restoration as a restoration type using exocad $\mathrm{GmbH}$ software (GmbH, Darmstadt, Germany) to create STL files and fabricate the crowns according to assigned groups, group A crowns were made using PMMA blocks (Whitepeaks Dental Solutions GmbH \& Co. $\mathrm{KG}$, Germany) using CAD/CAM system, and in group B, the crowns were made using 3D printable, biocompatible, 3D light-curing composite (GC Temp PRINT, Tokyo, Japan) fabricated via 3D dental printer DLP printing type.

A week later, the crowns were tried in and then cemented to selected teeth under isolation using resin cement (G-CEM LinkAce, GC Corporation, Tokyo, Japan) according to the manufacturers' instructions. Light curing was performed while applying pressure on the crown and then waiting for 5 minutes for complete cementing.

Evaluation of the crowns was assessed at that crown placement appointment then in the 3rd, 6th, and 12th months according to the "U.S. Public Health Service (USPHS) criteria ${ }^{16}$ by two independent investigators for crown retention rate (normal-small crack/fracture-large crack/fracture-complete loss of the crown). ${ }^{17}$ Gingival health was assessed using a periodontal probe. ${ }^{18}$ Marginal integrity was assessed by a dental probe (no catch- catch/no crevice visible/catch/ crevice visible/obvious crevice) ${ }^{19}$ ( - Fig. 2A, 2B, and 3A). The participants/parents and assessors/biostatisticians were blinded to the crowns' type (double-blinded study).

\section{Statistical Analysis}

The statistical analysis was performed using SPSS version 23 (Armonk, New York, United States), with a 95\% confidence interval and a $5.0 \%$ margin of error. Intraexaminer reproducibility for crowns' evaluation was calculated using Cohen's kappa test. The kappa for the intraexaminer agreement was found to be at an excellent agreement range of 0.97 .

Chi-square statistical test was used to compare the clinical outcomes between the two types of experimented crowns for "crowns' retention" evaluation at the end of the 12th-month follow-up times. Mann-Whitney's test was used to evaluate gingival health and marginal integrity for all follow-up periods, where the significance level was set at $p<0.05$.

\section{Results}

Fifty primary molars (maxillary and mandibular first and second primary molars) in 50 child patients were included in this study (-Table 1), 33 males and 17 females with a mean age of 6.04 years.

In all groups, clinical outcomes (crowns retentions, gingival health, and marginal integrity) scores were recorded for all crowns in the 3rd-, 6th-, and 12th-month follow-up periods.

For crown retention, chi-square statistical test was done (-Table 2, -Fig. 4); no statistically significant differences were noted at the end of follow-up times $(p=0.713)$.
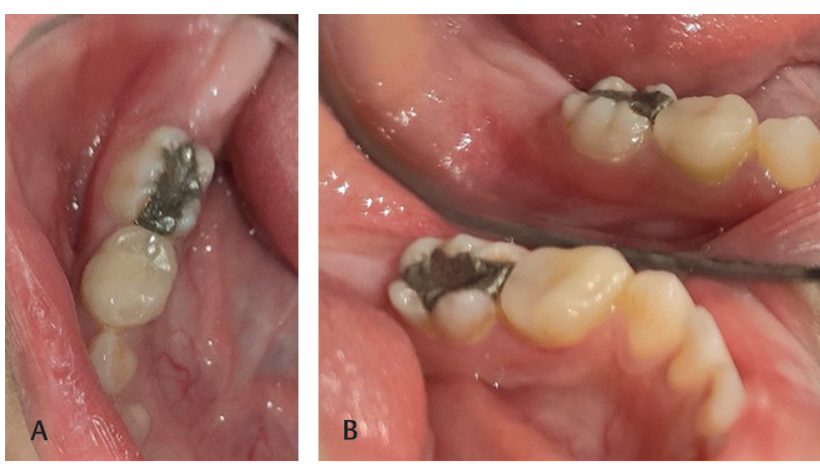

Fig. 2 (A, B) Polymethyl methacrylate crowns fabricated using CAD/ CAM after 12th-month follow-up. CAD/CAM, computer-aided design and manufacturing.
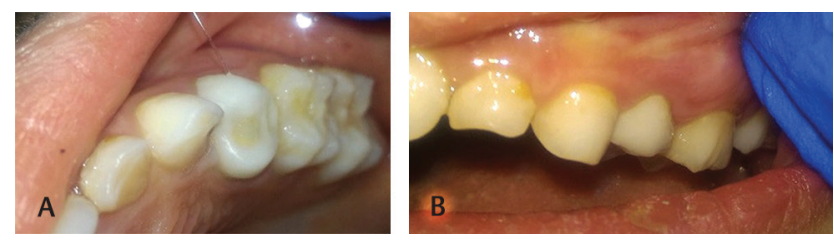

Fig. $3($ A, B) Photopolymer resin crowns fabricated using three-dimensional dental printer after 12th-month follow-up. 
Table 1 Patients age and gender

\begin{tabular}{|l|l|l|l|l|l|}
\hline & $N$ & Minimum & Maximum & Mean & SD \\
\hline Age & 50 & 4 & 8 & 6.04 & 1.06 \\
\hline & $N$ & Male & Female & & \\
\hline Gender & 50 & 33 & 17 & & \\
\hline
\end{tabular}

Abbreviation: SD, standard deviation.

Table 2 Crowns retention (restoration failure) evaluation

\begin{tabular}{|l|l|l|l|l|l|}
\hline $\begin{array}{l}\text { Crowns } \\
\text { retention }\end{array}$ & Baseline & 12 mo & $\%$ & $\begin{array}{l}\text { Chi- } \\
\text { square }\end{array}$ & $p$-Value \\
\hline CAD/CAM & 25 & 20 & $80 \%$ & 0.136 & 0.713 \\
\hline 3D printer & 25 & 21 & $84 \%$ & & \\
\hline
\end{tabular}

Abbreviation: CAD/CAM, computer-aided design and manufacturing.

Mann-Whitney's test was used for the evaluation of gingival health and marginal integrity between the two groups for the 3rd-, 6th-, and 12th-month follow-up times with no statistically significant differences noted at the 3rd- and 6th-month follow-ups ( $p=0.22$ and $p=0.084$ ), yet at the 12 th month, statistically significant differences were noted ( $p=0.022$ ) when comparing gingival health (-Table 3). However, no statistically significant differences were recorded between the two groups when comparing marginal integrity scores ( - Table 4 ) in all follow-up periods ( $p=0.504, p=450$, and $p=525)$.

The survival rate of 3D-printable crowns (group B) was 84\% compared with $80 \%$ survival rate in CAD/CAM fabricated crowns (group A) at the end of the 12th-month follow-up.

CAD/CAM fabricated crowns (group A) showed more gingival inflammation than 3D-printable crowns manufactured (group B) in all follow-up periods with a statistically significant difference in the 12th month.

Crown retention was clinically evaluated during follow-up periods by visual assessment, according to the USPHS, alpha criteria rating system. ${ }^{12}$

At the 3rd-month follow-up, three crowns were entirely lost in group A, while one crown was lost in group B. At the 6th month, another two crowns were wholly lost in group A, while no crowns were lost in group B. Finally, at the 12th month, a total of five crowns ( $80 \%$ success rate) were lost entirely in group $A$, while a total loss of four crowns in group $B$ ( $84 \%$ success rate) was recorded.

\section{Discussion}

This study was conducted to provide evidence-based treatment options for restoring pulp-treated primary molars with acceptable coast range when the most extensive caries risk development in patients left in poverty where the financial issue is essential with ethical appearance.

Esthetic management of pulpotomized primary molars is problematic for dentists despite the continuing development of dental materials. However, child behavior management, small teeth size, and lack of available esthetic choices for restoring primary molars make it challenging to manage. ${ }^{20}$

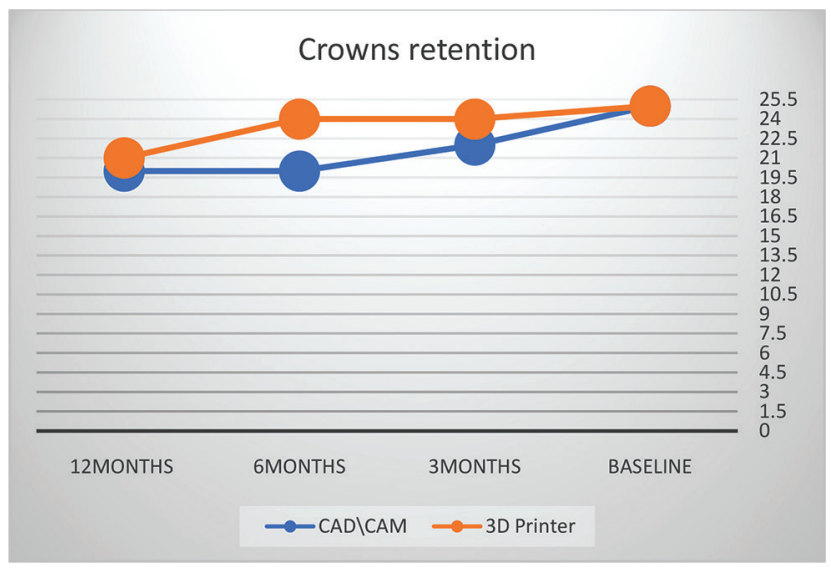

Fig. 4 Crowns retention for both groups for all follow-up times. CAD/ CAM, computer-aided design and manufacturing.

Moreover, using Exucade software provides ideal occlusal and proximal contact points and a better marginal fit at the gingival wall. ${ }^{21}$

In the dental field, clinical and laboratory research continues to evaluate printed materials for long-term intraoral use and good mechanical properties. ${ }^{22}$

After the 12th-month follow-up, the success rate of tested crowns (3D-printed composite-PMMA milled) for restoring posterior pulp-treated primary molars showed 84 and $80 \%$ success rates, respectively.

Furthermore, because of high fracture resistance for the two crown types, there was no noted chipping of crowns materials (large or small), and only (complete loss of crowns) results are shown as a final result.

Published data are limited regarding crowns fabricated using 3D dental printing systems. However, we know that adhesive-bonded restorations increase fracture load values compared with conventional luting of crowns. ${ }^{23-25}$ Our use of a 3D light-curing composite (GC Temp PRINT) material in fabricating crowns via 3D printer and cemented with an adhesive bonding system (resin cement, G-CEM LinkAce) displayed significant clinical stability in the 3D-printed composite crowns.

Gingival health evaluation in this study shows low gingival inflammation arising during the 3rd- to 6th-month follow-up visits and moderate with no statistical differences at the 12th-month follow-up. There was a better gingival response in 3D-printed crowns (group B) at the 12th-month follow-up. This result is likely from the manufacturing procedures for the crowns using 3D printing system with employs a self-heated tray and ultraviolet polymerization technique in the generation of crowns layer by layer to finally fabricate long-term provisional restoration for intraoral use with high biocompatible properties with a lower tendency of plaque build-up and thus a lower chance of gingival irritation. ${ }^{12}$ Moreover, because of this (low to moderate) gingival response in both groups, routine oral hygiene teaching is suggested to be added to the treatment plan. ${ }^{26}$

The microbial colonization of plaque on foreign restorative materials is the main reason for gingival inflammation. This plaque accumulation on the foreign restorative 
Table 3 Gingival health evaluation

\begin{tabular}{|c|c|c|c|c|c|c|c|c|c|}
\hline \multirow[t]{2}{*}{ Gingival health } & \multirow[t]{2}{*}{ Group } & \multicolumn{5}{|c|}{ Frequencies } & \multirow[t]{2}{*}{$Z$} & \multirow[t]{2}{*}{$p$-Value } & \multirow[t]{2}{*}{ Decision } \\
\hline & & No. & Mild & Moderate & Marked & Total & & & \\
\hline \multirow[t]{2}{*}{ Baseline } & CAD/CAM & 13 & 10 & 2 & 0 & 25 & \multirow[t]{2}{*}{0.000} & \multirow[t]{2}{*}{1.000} & \multirow[t]{2}{*}{ No differences } \\
\hline & 3D printer & 13 & 10 & 2 & 0 & 25 & & & \\
\hline \multirow[t]{2}{*}{$3 \mathrm{mo}$} & CAD/CAM & 6 & 12 & 4 & 0 & 22 & \multirow[t]{2}{*}{-1.226} & \multirow[t]{2}{*}{0.220} & \multirow[t]{2}{*}{ No differences } \\
\hline & 3D printer & 11 & 10 & 3 & 0 & 24 & & & \\
\hline \multirow[t]{2}{*}{$6 \mathrm{mo}$} & CAD/CAM & 3 & 12 & 5 & 0 & 20 & \multirow[t]{2}{*}{-1.727} & \multirow[t]{2}{*}{0.084} & \multirow[t]{2}{*}{ No differences } \\
\hline & 3D printer & 9 & 12 & 3 & 0 & 24 & & & \\
\hline \multirow[t]{2}{*}{$12 \mathrm{mo}$} & CAD/CAM & 0 & 14 & 6 & 0 & 20 & \multirow[t]{2}{*}{-2.287} & \multirow[t]{2}{*}{0.022} & \multirow[t]{2}{*}{ Statistical differences } \\
\hline & 3D printer & 6 & 12 & 3 & 0 & 21 & & & \\
\hline
\end{tabular}

Abbreviations: CAD/CAM, computer-aided design and manufacturing; 3D, three-dimensional. Note: Bold values signify test @<0.05 level.

Table 4 Marginal integrity evaluation

\begin{tabular}{|c|c|c|c|c|c|c|c|c|}
\hline \multirow{2}{*}{$\begin{array}{l}\text { Marginal } \\
\text { integrity }\end{array}$} & \multirow[t]{2}{*}{ Group } & \multicolumn{4}{|c|}{ Frequencies } & \multirow[t]{2}{*}{$Z$} & \multirow[t]{2}{*}{$p$-Value } & \multirow[t]{2}{*}{ Decision } \\
\hline & & Alpha & Bravo & Charlie & Total & & & \\
\hline \multirow[t]{2}{*}{ Baseline } & CAD/CAM & 25 & 0 & 0 & 25 & \multirow[t]{2}{*}{0.000} & \multirow[t]{2}{*}{1.000} & \multirow[t]{2}{*}{ No differences } \\
\hline & 3D printer & 25 & 0 & 0 & 25 & & & \\
\hline \multirow[t]{2}{*}{$3 \mathrm{mo}$} & CAD/CAM & 20 & 2 & 0 & 22 & \multirow[t]{2}{*}{-0.668} & \multirow[t]{2}{*}{0.504} & \multirow[t]{2}{*}{ No differences } \\
\hline & 3D printer & 23 & 1 & 0 & 24 & & & \\
\hline \multirow[t]{2}{*}{$6 \mathrm{mo}$} & CAD/CAM & 18 & 2 & 0 & 20 & \multirow[t]{2}{*}{-0.756} & \multirow[t]{2}{*}{0.450} & \multirow[t]{2}{*}{ No differences } \\
\hline & 3D printer & 23 & 1 & 0 & 24 & & & \\
\hline \multirow[t]{2}{*}{$12 \mathrm{mo}$} & CAD/CAM & 18 & 2 & 0 & 20 & \multirow[t]{2}{*}{-0.636} & \multirow[t]{2}{*}{0.525} & \multirow[t]{2}{*}{ No differences } \\
\hline & 3D printer & 20 & 1 & 0 & 21 & & & \\
\hline
\end{tabular}

Abbreviations: CAD/CAM, computer-aided design and manufacturing; 3D, three-dimensional.

materials depends on surface texture, surface area, surface smoothness, and microstructure. Therefore, the crowns made from photopolymer resin using a 3D-dental printer resulted in less plaque accumulation and gingival inflammation than crowns made from preparing ready-made PMMA blocks using CAD/CAM manufacturing. ${ }^{27,28}$

Another advantage of using 3D-printing systems (additive manufacturing approach) is material waste can be reduced by $40 \%$ in contrast to the milling-based technology using CAD/CAM technology. Therefore, 3D printing with additive manufacturing is considered to be more suitable comparing with milling technology. ${ }^{29}$

This study's limitations were: (1) crowns lost because of complete crown detachment and those crowns lost by inadvertent patient swallowing of that crown; therefore, recementing could not be done, and (2) the use of polyvinyl siloxane impressions as compared with digital scans for preparation acquisition. Future studies should pursue this digital methodology to determine its effect on outcomes.

\section{Conclusion}

3D-printed crowns and PMMA CAD/CAM crowns can be effectively used for restoring pulp-treated primary molars. Improved marginal adaption can be achieved thanks to digital design and printing/milling system accuracy, which results in a lower likelihood of cement washout, which reduces the possibility of cementation failure and subsequent decay.

Within the limitation of this random clinical trial, both CAD/CAM and 3D-printed crowns can be a reliable and effective choice for restoring primary molars. 3D-printed crowns performed better in the aspect of retention and gingival response.

\section{Ethical Approval}

Ethical approval was obtained from the Institutional Review Board of the lead authors' University (IRB Approval \#1191), and the trial was registered in the ANZCTR clinical registry (trial ID: ACTRN12619001650189).

\section{Authors' Contributions}

All the authors took part in conducting the study, manuscript preparation, and editing.

\section{Funding}

None.

\section{Conflict of Interest}

None declared.

\section{Acknowledgment}

This study has been performed at the Dental College at Damascus University, which deserves a great appreciation for all academic and financial support. 


\section{References}

1 Kindelan SA, Day P, Nichol R, Willmott N, Fayle SA; British Society of Paediatric Dentistry. UK National Clinical Guidelines in Paediatric Dentistry: stainless steel preformed crowns for primary molars. Int J Paediatr Dent 2008;18(Suppl 1):20-28

2 Bell SJ, Morgan AG, Marshman Z, Rodd HD. Child and parental acceptance of preformed metal crowns. Eur Arch Paediatr Dent 2010;11(5):218-224

3 US Department of Health and Human Services, 2000. National Institute of Dental and Craniofacial Research. Oral health in America: a report of the Surgeon General. Rockville (MD): US Department of Health and Human Services

4 Roberts JF. The open-face stainless steel crown for primary molars. ASDC J Dent Child 1983;50(4):262-263

5 Ram D, Fuks AB, Eidelman E. Long-term clinical performance of esthetic primary molar crowns. Pediatr Dent 2003;25(6):582-584

6 Abdulhadi B, Abdullah M, Alaki S, Alamoudi N, Attar M. Clinical evaluation between zirconia crowns and stainless steel crowns in primary molars teeth. J Pediatr Dent 2017;5:21-27. Doi:10.4103/jpd.jpd_21_17

7 Fazel S, Geddes JR, Kushel M. The health of homeless people in high-income countries: descriptive epidemiology, health consequences, and clinical and policy recommendations. Lancet 2014;384(9953):1529-1540

8 van Noort R. The future of dental devices is digital. Dent Mater 2012;28(1):3-12

9 Aiem E, Smaïl-Faugeron V, Muller-Bolla M. Aesthetic preformed paediatric crowns: systematic review. Int J Paediatr Dent 2017;27(4):273-282

10 Borges AFS, Correr GM, Sinhoreti MAC, Consani S, Sobrinho LC, Rontani RMP. Compressive strength recovery by composite onlays in primary teeth. Substrate treatment and luting agent effects. J Dent 2006;34(7):478-484

11 Collares K, Corrêa MB, Laske M, et al. A practice-based research network on the survival of ceramic inlay/onlay restorations. Dent Mater 2016;32(5):687-694

12 Tahayeri A, Morgan M, Fugolin AP, et al. 3D printed versus conventionally cured provisional crown and bridge dental materials. Dent Mater 2018;34(2):192-200

13 Schulz KF, Altman DG, Moher D; CONSORT Group. CONSORT 2010 Statement: updated guidelines for reporting parallel group randomised trials. BMC Med 2010;8:18

14 Frankl SN, Shiere FR, Fogels HR. Should the parent remain with the child in the dental operatory? J Dent Child 1962;29:150-163
15 Fuks AB, Ram D, Eidelman E. Clinical performance of esthetic posterior crowns in primary molars: a pilot study. Pediatr Dent 1999;21(7):445-448

16 Barnes DM, Blank LW, Gingell JC, Gilner PP. A clinical evaluation of a resin-modified. Glass ionomer restorative material. J Am Dent Assoc 1995;126(9):1245-1253

17 Salama AA. Zirconia crowns versus pre-veneered stainless steel crowns in primary anterior teeth a retrospective study of clinical performance and parental satisfaction. Egypt Dent J 2018;64(4):3075-3084 doi:10.21608/edj.2018.78283

18 Löe $\mathrm{H}$. The gingival index, the plaque index and the retention index systems. J Periodontol 1967;38(6):610-616

19 Atieh M. Stainless steel crown versus modified open-sandwich restorations for primary molars: a 2-year randomized clinical trial. Int J Paediatr Dent 2008;18(5):325-332

20 Shah PV, Lee JY, Wright JT. Clinical success and parental satisfaction with anterior preveneered primary stainless steel crowns. Pediatr Dent 2004;26(5):391-395

21 Mourouzis P, Arhakis A, Tolidis K. Computer-aided design and manufacturing crown on primary molars: an innovative case report. Int J Clin Pediatr Dent 2019;12(1):76-79

22 Abduo J, Lyons K, Bennamoun M. Trends in computer-aided manufacturing in prosthodontics: a review of the available streams. Int J Dent 2014;2014:783948

23 Mörmann WH, Bindl A, Lüthy H, Rathke A. Effects of preparation and luting system on all-ceramic computer-generated crowns. Int J Prosthodont 1998;11(4):333-339

24 Scherrer SS, de Rijk WG, Belser UC, Meyer J-M. Effect of cement film thickness on the fracture resistance of a machinable glass-ceramic. Dent Mater 1994;10(3):172-177

25 Bindl A, Lüthy H, Mörmann WH. Strength and fracture pattern of monolithic CAD/CAM-generated posterior crowns. Dent Mater 2006;22(1):29-36

26 Randall RC. Preformed metal crowns for primary and permanent molar teeth: review of the literature. Pediatr Dent 2002;24(5):489-500

27 Lee B-C, Jung G-Y, Kim D-J, Han J-S. Initial bacterial adhesion on resin, titanium and zirconia in vitro. J Adv Prosthodont 2011;3(2):81-84

28 Souza J, Mota R, Sordi MB, Passoni B, Benfatti C, Magini R. Biofilm formation on different materials used in oral rehabilitation. Brazilian Dental J 2016;27 (2):141-147

29 Azari A, Nikza S. The evolution of rapid prototyping in dentistry: A review. Rapid Prototyping J 2009;15(3):216-225 doi:10.1108/13552540910961946 